\title{
Caracterização do estro de novilhas cruzadas (Bos taurus indicus x Bos taurus taurus) por radiotelemetria
}

Claudia Maria BERTAN ${ }^{1}$ Mario BINELLI ${ }^{1}$ Ed Hoffmann MADUREIRA ${ }^{1}$

Correspondência para: ED HOFFMANN MADUREIRA Centro de Biotecnologia em Reprodução Animal

Departamento de Reprodução Animal Faculdade de Medicina Veterinária e Zootecnia

Universidade de São Paulo Av. Duque de Caxias Norte, 225 13630-000 - Pirassununga-SP e-mail:madureh@usp.br

Recebido para publicação: 29/07/2005 Aprovado para publicação: 13/02/2006

1 - Centro de Biotecnologia em Reprodução Animal do Departamento de Reprodução Animal da Faculdade de Medicina Veterinária e Zootecnia da Universidade de São Paulo, Pirassununga - SP

\section{Resumo}

Embora a técnica de inseminação artificial (IA) apresente inúmeras vantagens, no Brasil é empregada em apenas 7\% das fêmeas de corte. Falhas na detecção de estros constituem o principal fator limitante para a obtenção de êxito no emprego da técnica. Um sistema eletrônico, que se baseia na radiotelemetria, foi desenvolvido para a detecção de estros. Este sistema fornece o registro da data, horário e duração das montas ocorridas. O presente estudo teve como objetivo avaliar, pelo sistema de radiotelemetria, as características comportamentais de estro em novilhas cruzadas de corte (Bos taurus taurus $\mathrm{x}$ Bos taurus indicus), criadas em regime extensivo, na região sudoeste do Brasil. A hipótese testada foi que a duração dos estros e o número de montas são bastante variáveis entre as fêmeas e que a maioria das montas ocorre no período noturno. Para a sincronização dos estros as novilhas receberam $0,5 \mathrm{mg}$ de MGA/cabeça/dia, uma vez ao dia, durante 8 dias, e uma injeção de $15 \mathrm{mg}$ de luprostiol (PG) via IM no último dia da ingestão de MGA. Os estros foram registrados pelo sistema de radiotelemetria "Heat-Watch", durante um período de até 120 horas após a aplicação de PG. A duração média dos estros foi de 10,4 \pm 5,7 horas, duração que variou de 45 minutos a 22,7 horas. O número médio de montas foi de $26,2 \pm 13,6$ e variou de 3 a 81 montas. A duração média das montas foi de 2,7 $\pm 0,3$ segundos. Dransfield et al. ${ }^{1}$ classificaram os estros em curta ( $<7$ horas) e longa ( $\geq 7$ horas $)$ duração e baixa ( $<1,5$ montas/hora) e alta $(\geq 1,5$ montas/hora) intensidade. Houve uma maior incidência dos estros de longa duração quando comparados aos de curta duração $(72,8 \%$ vs. $27,2 \% ; \mathrm{P}<0,05)$ e uma maior incidência dos estros de alta intensidade quando comparados aos de baixa intensidade $(70,2 \%$ vs. $29,8 \%$; $<<0,05)$. A média geral de montas diurnas foi de 10,0 \pm 9,7 (das 7:00 às 19:00 horas) e de montas noturnas de 13,0 \pm 12,4 (das 19:00 às 7:00 horas), sendo que não foi observado efeito de período do dia no número de montas $(\mathrm{P}=0,08)$. No presente estudo confirmou-se a hipótese de que o comportamento de estro é extremamente variável entre as fêmeas, mas não a de que a maioria das montas ocorre no período noturno.

\section{Introdução}

Na pecuária de corte a produtividade está diretamente relacionada a eficiência reprodutiva das fêmeas. As fêmeas devem apresentar um adequado intervalo entre partos, ao redor de 12 meses, o que resulta na produção de um bezerro/vaca/ano. No Brasil a taxa média de natalidade é de $60 \%$ e a de mortalidade dos bezerros até a desmama é de $8 \%$, taxas que associadas caracterizam um intervalo entre partos de 
24 meses e a produção de 0,5 bezerro/vaca/ ano.

O aumento dos índices reprodutivos deve estar sempre aliado a melhoria genética dos produtos nascidos. Melhores índices zootécnicos poderiam ser alcançados pelo emprego da técnica de inseminação artificial (IA). O acasalamento programado de fêmeas azebuadas com reprodutores de raças européias tem demonstrado inúmeras vantagens nos produtos nascidos, dentre as quais: aumento na velocidade de crescimento e diminuição da idade ao abate, melhoria no rendimento e qualidade de carcaça, precocidade sexual, fertilidade e grande habilidade materna nas fêmeas. Embora as vantagens oferecidas pelo uso da técnica de IA sejam conhecidas e o mercado nacional de IA tenha apresentado crescimento nos últimos anos, apenas 7\% das fêmeas de corte são inseminadas no Brasil. Em fêmeas de corte criadas em regime extensivo, as falhas na detecção de estros constitui o principal fator limitante do sucesso no emprego da técnica de IA. Deve-se considerar que cada estro não detectado representa à fêmea um aumento no intervalo entre partos em média de 21 dias.

Embora muitos métodos de detecção de estros tenham sido desenvolvidos nos últimos anos, o método mais utilizado nos programas de IA ainda é a observação visual realizada da maneira convencional. Este método propõe a realização diária de duas observações, uma no início da manhã e outra ao final da tarde com, no mínimo, um hora de duração. Entretanto, Senger ${ }^{2}$ relata que o tempo total em que os animais permanecem montados durante o estro corresponde a menos de $1 \%$ da duração total dos estros, característica que dificulta a detecção de tal evento fisiológico. Em rebanhos leiteiros, a observação convencional é responsável por falhas de até mais de $50 \%$ na identificação de estros. Estudos complementares avaliaram as concentrações de progesterona $\left(\mathrm{P}_{4}\right)$ no leite durante a IA, e constataram que $22 \%$ das fêmeas falharam em conceber porque eram inseminadas em momento inadequado, sendo que 5 a $30 \%$ delas foram inseminadas na ausência de estro ${ }^{3}$ Muitos métodos foram desenvolvidos na tentativa de superar falhas na detecção dos estros, como buçais marcadores em fêmeas androgenizadas ou rufiões, vídeo-câmeras, cães treinados para detectar odores relacionados ao estro, pedômetros e outros. Alguns destes métodos apresentam eficiência na detecção, desde que utilizados simultaneamente com a observação visual ${ }^{1,2,4}$; Dransfield $^{1}$; Senger ${ }^{2}$. A tecnologia eletrônica possibilitou o desenvolvimento de técnicas automáticas na detecção de estros. Dinsmore e Cattell ${ }^{5}$ desenvolveram um sistema eletrônico para a detecção de estros através da radiote-lemetria, denominado de "HeatWatch". Esse sistema é composto por transmissores sensíveis à pressão, fixados na região do sacro da fêmea. Quando acionados pela pressão gerada na atividade de monta, emitem sinais de radiofreqüência que são captados por uma antena em um raio de aproximadamente 450 metros. Os sinais são recebidos e arma-zenados em um dispositivo, que transfere as informações a um "software" capaz de organizar todas as informações de estros referentes a cada animal contemplando data, hora e duração das montas, na forma de tabelas e gráficos. Stevenson et al. compararam a eficiência deste sistema com o método tradicional de observação visual na detecção de estros em novilhas sincronizadas, e observaram uma acurácia de $73 \%$ na observação visual e de $100 \%$ no método de radiotelemetria. Neste estudo, do total de 41 animais em estro, apenas 30 fêmeas foram observadas da maneira convencional. Das 11 fêmeas detectadas exclusivamente pelo método de radiotelemetria, e não observadas da maneira convencional, cinco apresentaram um comportamento de estro caracterizado por cinco ou menos montas. Xu et al. ${ }^{7}$ confirmaram a acurácia de $100 \%$ no método de radiotelemetria e registraram ao mesmo uma eficiência de $91,7 \%$. O sistema "HeatWatch" fornece, com exatidão, os horários de início e de final dos estros, os horários 
das montas, o número de montas e a sua duração tornando possível avaliar, com detalhes, as características comportamentais de estro das fêmeas bovinas.

Quando Hurnik et al. ${ }^{8}$ realizaram uma observação contínua em rebanhos leiteiros através de câmeras de vídeo durante 80 dias, observaram que $70 \%$ das montas ocorreram no período noturno. Ainda, Helmer e Britt ${ }^{6}$ constataram que do total de fêmeas em estro, $100 \%$ aceitaram monta pela manhã e apenas $77,77 \%$ foram montadas pela tarde. Os autores sugerem que a menor temperatura da manhã favoreceria a atividade de monta visto que, em observacões realizadas às 12 horas, horário do dia em que a temperatura ambiente é mais elevada, apenas $44,44 \%$ das fêmeas em estro foram montadas.

Considerando as diferentes características comportamentais de estro, Dransfield et al. ${ }^{1}$ caracterizaram os estros em diferentes categorias, considerando sua duração e intensidade. Quanto à duração, podem apresentar curta ( $<7$ horas de duração) ou longa duração ( $\geq 7$ horas de duração). Quanto à intensidade, podem ser de baixa $(<1,5$ montas/hora) ou de alta intensidade $(\geq 1,5$ montas/hora).

Baseando-se nos dados descritos na literatura pode-se considerar que as diferentes características de estro entre os animais podem contribuir de maneira importante na baixa eficiência da detecção de estros no sistema de observação convencional. Assim, o presente estudo teve como objetivo avaliar as características comportamentais de estro, pelo sistema de radiotelemetria mais conhecido como "Heat-Watch", em novilhas de cruzadas de corte (Bos taurus taurus x Bos taurus indicus), criadas em regime extensivo na região sudoeste do Brasil. A hipótese é que a duração dos estros e o número de montas são bastante variáveis entre as fêmeas bovinas e que a maioria das montas ocorrem no período noturno.

\section{Materiais e Métodos}

Local do Experimento e Animais

O experimento foi realizado no Centro de Biotecnologia em Reprodução Animal, na Faculdade de Medicina Veterinária e Zootecnia da Universidade de São Paulo. Foram utilizadas 150 novilhas mestiças de corte (Bos taurus indicus $\times$ Bos taurus taurus), entre 22 e 33 meses. Para que as características comportamentais de estro fossem analisadas, as novilhas tiveram os estros sincronizados. No dia do início do tratamento para a sincronização dos estros as novilhas foram pesadas e avaliadas quanto à condição corporal, segundo a classificação de Richards et al. ${ }^{9}$ A média e o desvio padrão das novilhas foram de $376,4 \pm 53,6$ para o peso e 4,8 \pm 0,3 para a condição corporal. Durante a execução do experimento as fêmeas foram mantidas em piquetes, com água à disposição. A alimentação foi baseada no pastejo (Brachiaria decumbens var. marandu). A suplementação mineral foi associada ao suplemento proteíco.

\section{Delineamento Experimental}

O delineamento experimental utilizado foi o inteiramente casualizado, onde se considerou a vaca como unidade experimental. Antes do início da sincronização dos estros, as novilhas foram avaliadas quanto à normalidade do sistema genital e das condições ovarianas, utilizando-se um aparelho de ultra-som (Aloka Ultrasound Diagnostic Equipament, Modelo SSD-500, Tokyo/Japão) com transdutor linear de 5 MhZ. Os estros foram sincronizados pela administração do acetato de melengestrol (MGA) fornecido aos animais adicionado ao suplemento mineral protéico. As novilhas foram submetidas a um período de adaptação, durante os 7 dias que antecederam o início da ingestão do MGA, no qual o sal protéico (Frepal Salutti Complexos Minerais) foi fornecido na mesma quantidade e nas mesmas condições que seria oferecido durante o tratamento, porém não adicionado de MGA. Todas as novilhas receberam suplemento com $0,5 \mathrm{mg}$ de MGA (The Upjohn Company) em $200 \mathrm{~g}$ de suplemento/cabeça/dia, uma vez ao dia, durante oito dias, e uma injeção 
intramuscular (IM) de $15 \mathrm{mg}$ de luprostiol (PG; ProsolvinÒ, Intervet) no último dia da ingestão de MGA. Imediatamente após a aplicação de PG, as observações de estros foram realizadas continuamente durante um período de até 120 horas, para tanto se utilizou o sistema de radiotelemetria designado de "Heat-Watch" (DDx Incorporated, Denver, Colorado/EUA). Do total de 150 fêmeas tratadas para a sincronização dos estros, 77 novilhas tiveram os estros sincronizados no período de até 120 horas após a aplicação de PG. Assim, no presente estudo as características de estros foram avaliadas em 77 novilhas. Após a administração de PG, as novilhas foram colocadas na presença de rufiões na proporção de um rufião para 25 fêmeas. Considerou-se o início do estro quando ocorreu a aceitação de pelo menos três montas, com duração $\geq 2$ segundos, em um intervalo de 2 horas. Considerou-se o final do estro o momento da última monta desde que esta tivesse ocorrido no máximo 4 horas após a penúltima. Os estros foram caracterizados segundo Dransfield et $\mathrm{al}^{1} \mathrm{~m}$ estros de curta ( $<7$ horas) e longa $(\geq 7$ horas) duração e baixa $(<1,5$ montas/hora) e alta $(\geq 1,5$ montas/hora) intensidade.

\section{Análise Estatística}

Os resultados foram analisados estatisticamente empregando-se os programas computacionais Statistical $5.0^{10} \mathrm{e}$ Statistical Analysis System. ${ }^{11}$ Foram analisadas às médias e o desvio padrão das características: duração dos estros, número de montas e duração das montas. A variável porcentagem de novilhas segundo a categoria de estros (classificação de Dransfield et al. ${ }^{1}$ ) foi analisada pelo teste de Qui-quadrado. ${ }^{12}$ A média do número de montas diurnas e noturnas foi submetida à análise de variância. Para as análises considerou-se um nível de significância de $5 \%$.

\section{Resultados e Discussão}

Conforme representado na tabela 1 , no presente experimento a duração média dos estros foi de 10,4 \pm 5,7 horas (h), duração que variou de 45 minutos a 22,7 h. Em outros estudos, que utilizaram o sistema de radiotelemetria em rebanhos leiteiros, relataram-se durações de estros em média de $12,1 \mathrm{~h}^{13} ; 9,5 \mathrm{~h}^{14} ; 14,0 \pm 0,8 \mathrm{~h}^{6} ; 8,6 \mathrm{~h}^{7} \mathrm{e}$ $7,1 \pm 5,4 \mathrm{~h}$, duração que variou de 33 minutos a $35,8 \mathrm{~h}^{1}$

Em estudos que utilizaram o sistema de radiotelemetria em novilhas cruzadas, a duração dos estros foi de e 9,38 $\pm 0,7 \mathrm{~h}^{15} \mathrm{e}$ $9,43 \mathrm{~h}$ sendo de $8,52 \pm 1,2 \mathrm{~h}$ para novilhas Angus, 6,65 $\pm 1,2 \mathrm{~h}$ para novilhas Brahma e $11,90 \pm 1,2 \mathrm{~h}$ para novilhas cruzadas, duração que variou de 0,23 a 27,3 h. ${ }^{16}$ No presente estudo a média observada na duração dos estros foi semelhante às observadas nos outros estudos realizados com novilhas cruzadas.

Deve-se considerar relevante que, no presente estudo e em outros estudos que utilizaram a radiotelemetria ${ }^{1,6,15}$, a duração dos estros caracterizou-se pode ser bastante variável. Stevenson et al..$^{20}$ relataram que a duração dos estros variou de 2,6 a 26,2 h, sendo que em 20,5\% das novilhas os estros duraram menos que $10 \mathrm{~h}$ e em $50 \%$ menos de $6 \mathrm{~h}$. No presente estudo, verificou-se que $49,35 \%$ das novilhas apresentaram estros com duração menor que 10 horas. Deve-se considerar que no sistema convencional normalmente os estros são observados durante $1 \mathrm{~h}$ a cada intervalo de $10 \mathrm{~h}$. Considerando tal característica deste sistema e a alta porcentagem de animais com estros com duração menor que 10 h (49,35\%) verificados no presente estudo, pode-se supor um comprometimento da eficiência do sistema convencional na detecção de fêmeas em estro.

Utilizando a classificação proposta por Dransfield et al. ${ }^{1}$, constatou-se no presente estudo que $27,3 \%$ dos estros foram caracterizados como de curta duração (duraram menos que 7 horas) e $72,7 \%$ de longa duração (duraram mais que 7 horas). Observação muito semelhante foi relatada por Rocha ${ }^{2}$, quando verificou que $27 \%$ das novilhas apresentaram estros de curta 
duração e $73 \%$ de longa duração. Estes dados diferem dos verificados por Dransfield et al. ${ }^{1}$ em rebanhos leiteiros, quando observaram que $58,4 \%$ das fêmeas apresentaram estros de curta duração e $41,6 \%$ de longa duração. Relata-se na literatura vários fatores que poderiam interferir na duração dos estros, dentre os principais encontram-se variações ambientais, nutrição, estágio de produção e tipo de piso existente no local onde as montas são realizadas ${ }^{17,18,19,20}$.

No presente estudo verificou-se um número médio de montas de 26,2 $\pm 13,6$, número que variou de 3 a 81 montas. As montas tiveram uma duração média de 2,7 $\pm 0,3$ segundos (Tabela 1). Deve-se ressaltar que embora o número de montas tenha sido bastante variável a duração no tempo de monta foi caracterizado por uma baixa variação. Hurnik et al. ${ }^{8}$ relacionaram grande variabilidade no número de montas ao número de fêmeas em estro. Quando uma, duas ou três fêmeas apresentaram estros simultâneamente o número de montas foi de 11,2,36,6 e 52,6, respectivamente. Helmer e Britt ${ }^{19}$ confirmaram tal observação quando verificaram em novilhas leiteiras, uma média de 2,3 montas quando havia uma fêmea em estro e 6,1 montas quando mais de uma fêmea em estro. Considerando que no presente estudo as novilhas foram tratadas para a sincronização dos estros, o grande número de fêmeas em estro simultaneamente certamente contribuiu para um incremento no número de montas. Deve-se considerar que o emprego de protocolos de sincronização de estros podem contribuir no aumento da eficiência na detecção de fêmeas em estro pelo sistema convencional, embora seja uma tendência atual, a sincronização dos estros associada à sincronização da ovulação, estratégia que torna desnecessária a detecção de estros.

O número de montas detectado no presente experimento foi menor do que os relatados na literatura de 36,6 a $52,6^{8}$ e 50,1 \pm 6,46 $; 19 \pm 3,6$ para novilhas Angus, $25 \pm$ 5,4 para novilhas Brahma e $37 \pm 5,5$ para cruzadas, contabilizando uma média geral de $27 \pm 2,9$ montas, embora tenha variado de 3 a 111 montas. ${ }^{15}$ A maior média relatada por Stevenson et al. ${ }^{6}$ provavelmente ocorreu porque os autores consideraram montas com duração $\geq 1$ segundo, enquanto no ppresente estudo considerou-se apenas montas com duração $\geq 2$ segundos. Contudo, as médias observadas no presente estudo foram maiores do que as descritas em fêmeas leiteiras quando apenas 6 montas excederam 2 segundos ${ }^{14}$; 6,1 montas com mais de uma fêmea em estro ${ }^{19} ; 8,8^{21} ; 12,8 \pm$ 9,9 considerando montas $\geq 2$ segundos $^{1} \mathrm{e}$ de 11,2 montas 7 . Deve-se ressaltar que estes trabalhos não podem ser comparados de maneira absoluta considerando o grande número de fatores que podem interferir no comporta-mento de monta. Helmer e Britt $^{19}$ ainda relatam que fêmeas mantidas em piso de terra normalmente realizam um maior número de montas quando comparadas às colocadas em piso de concreto. Este fato provavelmente tenha privilegiado o número de montas nas fêmeas de corte que tiveram os estros observados em piquetes com o piso de terra. A duração média das montas e a baixa variabilidade no tempo de monta observada no presente estudo, foram semelhantes às relatadas na literatura. Reporta-se durações médias de 2,5 segundos ${ }^{7}, 2,53$ a 2,83 segundos ${ }^{22}$ e 3,1 a 3,6 segundos ${ }^{23}$.

Senger ${ }^{2}$ relata que o tempo total em que os animais permanecem montados durante o estro corresponde a menos de $1 \%$ da duração total dos estros, comportamento que dificulta a detecção de tal evento fisiológico. De maneira geral, considerando que no presente estudo os estros duraram em média 10,4 horas, período em que o número de montas foi de 26,2 com uma duração média de 2,7 segundos, o período total de atividade de monta corresponderia a 1,3 minutos, o que representa $0,18 \%$ da duração total dos estros, ou seja, muito menos de $1 \%$. Deve-se considerar ainda que no presente estudo, o número de montas possivelmente tenha sido contemplado pelo 
grande número de fêmeas em estro simultâneamente. Assim, em novilhas observadas da maneira convencional o período de tempo de monta em relação a duração do estro possivelmente é menor que $0,18 \%$, fato que certamente compromete a ineficiência da detecção de estros nas observações realizadas.

Dransfield et al. ${ }^{1}$ distribuíram os estros em diferentes categorias, considerando sua duração e intensidade. Quanto à duração, podem apresentar curta ( $<7$ h de duração) ou longa duração ( $\geq 7 \mathrm{~h}$ de duração). Quanto à intensidade, podem ser de baixa $(<1,5$ montas/hora) ou de alta intensidade $(\geq 1,5$ montas/hora). Os autores observaram 2055 fêmeas leiteira e constataram que $24,1 \%$ dos estros foram de baixa intensidade e curta duração (BICD); 33,2\% baixa intensidade e longa duração (BILD); 34,3\% alta intensidade e curta duração (AICD) e 8,4 \% alta intensidade e longa duração (AILD). Conforme representado na tabela 1 , no presente estudo observou-se uma maior incidência dos estros de alta intensidade quando compara-dos aos de baixa intensidade $(70,2 \%$ vs. $29,8 \% ; \mathrm{P}<0,05)$. Também verificou-se uma maior incidência dos estros de longa duração quando comparados aos de curta duração $(72,8 \%$ vs. $27,2 \% ; \mathrm{P}<0,05)$. No presente estudo $6,5 \%$ dos estros foram caracterizados como de BICD; 23,4\% BILD; $20,8 \%$ de AICD e 49,4\% de AILD, observações que diferem das descritas por Dransfield et al. ${ }^{4}$ Deve-se considerar que no rebanho leiteiro houve uma menor incidência de estros de AILD e que o oposto foi verificado no presente estudo realizado em novilhas de corte. Esta verificação também foi realizada por

Tabela 1 - Média e desvio padrão da duração dos estros, número de montas, duração das montas e porcentagem de estros segundo classificação de Dransfield et al. ${ }^{4}$, em novilhas cruzadas (Bos taurus indicus x Bos taurus taurus) que tiveram os estros sincronizados

\begin{tabular}{|c|c|c|}
\hline tica Avaliada & Média + Desvio Padrão & Porcentagem (Proporção) \\
\hline Duração dos estros ${ }^{\mathrm{a}}$ & $10,4 \pm 5,7$ horas & \\
\hline Número de montas ${ }^{b}$ & $26,2 \pm 13,6$ montas & \\
\hline Duração das montas ${ }^{c}$ & $2,7 \pm 0,3$ segundos & \\
\hline Estros de alta intensidade ${ }^{d}$ & & $70,2 \%(54 / 77)$ \\
\hline Estros de baixa intensidade $\mathrm{e}^{\mathrm{e}}$ & & $29,8 \%(23 / 77)$ \\
\hline Estros de longa duração ${ }^{\dagger}$ & & $72,8 \%(56 / 77)$ \\
\hline Estros de curta duraçãog & & $27,2 \%(21 / 77)$ \\
\hline $\begin{array}{l}\text { Estros de baixa intensidade e curta } \\
\text { duração } \mathrm{O}^{\mathrm{h}}\end{array}$ & & $6,5 \%(5 / 77)$ \\
\hline $\begin{array}{c}\text { Estros de baixa intensidade e longa } \\
\text { duraçãoi }\end{array}$ & & $23,4 \%(18 / 77)$ \\
\hline $\begin{array}{c}\text { Estros de alta intensidade e curta } \\
\text { duração }\end{array}$ & & $20,8 \%(16 / 77)$ \\
\hline $\begin{array}{c}\text { Estros de alta intensidade e longa } \\
\text { duração }\end{array}$ & & $49,4 \%(38 / 77)$ \\
\hline
\end{tabular}

aPeríodo compreendido entre a primeira monta que caracteriza o início do estro, e, a última que determina o final do estro;

bNúmero de montas com uma duração mínima de 2 segundos;

"Duração das montas ocorridas no período de estro;

'Estros com uma média maior ou igual a 1,5 montas/hora;

eEstros com uma média menor que 1,5montas/hora;

'Estros com uma duração maior ou igual a 7 horas;

¿Estros com uma duração menor que 7 horas;

"Estros com uma média menor que 1,5 montas/hora, com uma duração menor que 7 horas;

'Estros com uma média menor que 1,5 montas/hora, com uma duração maior ou igual a 7 horas;

'Estros com uma média maior ou igual 1,5 montas/hora, com uma duração menor que 7 horas;

KEstros com uma média maior ou igual 1,5 montas/hora, com uma duração maior ou igual a 7 horas: 
Rocha ${ }^{22}$ quando observou em novilhas de corte a ocorrência de $11,2 \%$ de estros de BICD; $10,1 \%$ de BILD; $15,7 \%$ de AICD e $62,9 \%$ de AILD. Pode-se supor que fêmeas leiteiras e de corte apresentam características comportamentais de estros bastante diferenciadas quanto à duração do estro e número de montas realizadas, onde a duração dos estros e o número de montas seria maior em fêmeas de corte.

Muitos estudos ilustram diferentes aspectos que poderiam interferir de maneira importante nas características de estro determinando variações no número e na duração das montas. Helmer e Britt ${ }^{19}$ demonstraram que $67 \%$ das montas são realizadas por outras fêmeas em estro e $20 \%$ por fêmeas em proestro. Os autores ainda relatam que a presença de fêmeas na fase luteal e prenhes no lote de observação de estros podem contribuir para minimizar as atividades de monta do rebanho. Vailes e Britt ${ }^{24}$ demonstraram que a atividade de monta em fêmeas leiteiras pode aumentar em até quatro vezes quando as mesmas são colocadas em piso de terra, em relação àquelas mantidas em piso de concreto.

No presente estudo a média geral de montas diurnas foi de 10,0 \pm 9,7 (das 7:00 às 19:00 horas) e de 13,0 $\pm 12,4$ para as montas noturnas (das 19:00 às 7:00 horas). Não foi observado efeito de período do dia no número de montas $(\mathrm{P}=0,08)$. Hurnik et al. ${ }^{8}$ através do uso de câmeras de vídeo, observaram que em rebanhos leiteiros $70 \%$ das montas ocorreram no período noturno, resultado não confirmado no presente estudo, considerando que $43,6 \%$ das montas ocorreram durante o dia e $56,4 \%$ à noite.
Dados semelhantes ao do presente estudo foram relatados por Stevenson et al. ${ }^{20}$, que verificaram $54 \%$ de montas diurnas e $46 \%$ de noturnas.

Gwazdauskas et al. ${ }^{18}$ observaram a influência da temperatura ambiente no número de montas em temperatura. Os autores relatam uma correlação positiva entre o aumento da temperatura ambiente e o número de montas realizadas em uma faixa de temperatura entre 25 e $30^{\circ} \mathrm{C}$, entretanto, em temperaturas acima de $30^{\circ} \mathrm{C}$ caracterizouse um decréscimo no número de montas. De fato, Helmer e Britt ${ }^{19}$ verificaram que do total de animais em estro, $100 \%$ das fêmeas aceitaram a monta pela manhã, $77,8 \%$ pela tarde e apenas $44,5 \%$ no meio do dia.

O presente trabalho não teve como objetivo avaliar a eficiência da radiotelemetria na detecção de estros, entretanto alguns estros identificados pelo sistema possivelmente não seriam detectados pela maneira convencional, considerando que $18,2 \%$ dos estros foram de curta duração e ocorreram durante a madrugada.

No presente estudo confirma-se a hipótese de que o comportamento de estro é extremamente variável entre as fêmeas entretanto a maioria das montas não ocorrem no período noturno.

\section{Agradecimentos}

Os autores agradecem à Fundação de Amparo a Pesquisa do Estado de São Paulo (FAPESP): Processo 97/09140-5, à Salluti, Merial Saúde Animal Ltda e à Prefeitura do Campus Administrativo de Pirassununga (PCAPS)

\section{Estrous characterization in cross-bred heifers (Bos taurus indicus x Bos taurus taurus) by radiotelemetry}

\section{Abstract}

Although artificial insemination (AI) has countless advantages, in Brazil this technique is performed in only $7 \%$ of beef cattle. Failures on estrous detection are the main limiting factor to the success of this technique. An electronical system, based on radiotelemetry, was developed for estrous detection. This system provides day, hour and
Key-words:

Estrous.

Radiotelemetry. Heat-Watch. 
service length. The present study aimed to evaluate by radiotelemetry system the behavioral characteristics of the estrous of crossbred heifers (Bos taurus taurus $\mathrm{x}$ Bos taurus indicus) raised at pasture regimen in southwest

\section{Referências}

1 DRANSFIELD, M. G. B. Timing of insemination of dairy cows identified in estrus by a radiotelemetric estrus detection system. Journal of Dairy Science, v. 81, n. 7, p.1874-1882, 1998.

2 SENGER, P.L. The estrus detection problem: new concept, technologies, and possibilites. Journal of Dairy Science, v. 77, n. 9, p. 2745-2753, 1994

3 BARR, H. L. Influence of estrous detection on open in dairy herds. Journal of Dairy Science, v. 58, n. 2, p. 246-247, 1975

4 STEVENSON, J.S.; BRITT, J. H. Detection of estrus by three methods. Journal of Dairy Science, v. 60, n. 12, p.1994-1998, 1977.

5 DINSMORE, R. P.; CATTEL, M. B. Field trial of a radiotelemetry estrous detection system. Journal of Dairy Science, v. 76, p. 227, 1993. Suplemment 1.

6 STEVENSON, J. S.; et al. Detection of estrus by visual observation and radiotelemetry in peripubertal, estrussynchronized beef heifers. Journal of Animal Science, v.7 4, n. 4, p. 729-735, 1996

$7 \mathrm{XU}, \mathrm{Z} . \mathrm{Z}$. et al. Estrus detection using radiotelemetry or visual observation and tail painting for dairy cows on pasture. Journal of Dairy Science, v.81, n.11, p. 2890-2896, 1998

8 HURNIK, J. F.; KING, G. J.; ROBERTSON, H. A. Estrous and related behaviour in postpartum Holstein cows. Applied Animal Ethology, v. 2, p. 55-68, 1975. 9 RICHARDS, M. W.; SPITZER, J. C.; WARNER, M.B. Effect of varying levels of postpartum nutrition and body condition at calving on subsequent reproductive performance in beef cattle. Journal of Animal Science, v. 62, n. 2, p. 300-306, 1986.

10 STATISTICA version 5.0. Tulsa: Copyrightâ Satsoft Inc, 1984-1985

11 SAS Institute. SAS user's guide statistics 89. Raleigh: SAS, 1989.

12 SEIGEL, S. Estatística não paramétrica. São Paulo: McGraw-Hill, 1975. 305 p.

13 NEBEL, R. L.; et al. Radiotelemetered measures of mounting activity for detection of estrus in lactating dairy cows. Journal of Dairy Science, v. 75, p. 242, 1992. Supplement 1

14 WALKER, W. L.; NEBEL, R. L.; MCILLARD, M. L. Time of ovulation relative to mounting activity in dairy cattle. Journal of Dairy Science, v. 79, n. 9, p. 15551561, 1996

15 RAE, D. O.; et al Assessment of estrus detection by visual observation and eletronic detection methods and characterization of factors associated with estrus and pregnancy in beef heifers. Theriogenology, v. 51 n. 6, p. 1121-1132, 1999.

16 ROCHA, J. L.; MADUREIRA, E. H.; BARNABE, R. C. Características do estro e da ovulação em novilhas de corte mestiças sincronizadas e em novilhas e vacas Nelore com estros naturais, detectadas através do Sistema "HEAT-WATCH". Revista Brasileira de Reprodrução Animal Anim., v.23, n.3, p.162-164, 1999.

17 BRITT, J. H.; et al. Determinants of estrous behavior in lactating Holstein cows. Journal of Dairy Science v.69, n.8, p. 2195-2202, 1986.

18 GWAZDAUSKAS, F. C.;LINEWEAER,J. A. MCGILLIARD, M. L. Environmental and management factors affection estrous activity in dairy cattle. Journal of Dairy Science, v. 66, n. 7, p.1510-1514, 1983.

19 HELMER, S.D.; BRITT, J. H. Mounting behavior as affected by stage of estrous cycle in Holstein heifers. Journal of Dairy Science, v. 68, n. 5, p.1290-1296, 1985.

20 LUCEY, S. G.; ROWLANDS, G. J.; RUSSEL, A. M The association between lameness and fertility in dairy cows. Veterinary Record, v. 118, n. 7, p. 628-634, 1986.

21 WALTON, J. S.; VEENHUIZEN, L. P.; KING, G. J. Relation-ships between time of day, estrous behavior and the preovulatory luteinizing hormone surge in Holstein cows after treatment with cloprostenol. Journal of Dairy Science, v. 70, n. 8, p.1652-1663 1987.

22 ROCHA J. L. Sistema "Heat-Watch" para controle do ciclo estral de novilhas de corte mestiças europeu x Nelore (Bos taurus $x$ Bos indicus): sincronização hormonal do estro e das ondas foliculares. São Paulo, 2000. 130p. Tese (Doutorado)-Faculdade de Medicina Veterinária e Zootecnia, Universidade de São Paulo. (No prelo)

23 STEVENSON, J. S. et al. Luteolysis during two stages of the estrous cycle: subsequent endocrine profiles associated with radiotelemetrically detected estrus in heifers. Journal of Dairy Science, v. $81, \mathrm{n}$. 11 , p. 2897-2903, 1998

24 VAILES, L. D.; BRITT, J. H. Influences of footing surface on mounting and other sexual behaviors of estrual Holstein cows. Journal of Animal Science v.68, n. 8, p. 2333-2339, 1990 